\title{
Tubular microdiscectomy: techniques, complication avoidance, and review of the literature
}

\author{
Aaron J. Clark, MD, PhD, ${ }^{1}$ Michael M. Safaee, MD, ${ }^{1}$ Nickalus R. Khan, MD, ${ }^{2}$ Matthew T. Brown, MD, ${ }^{2}$ \\ and Kevin T. Foley, MD2,3
}

1Department of Neurological Surgery, University of California, San Francisco, California; ${ }^{2}$ Department of Neurosurgery, University of Tennessee Health Science Center; and ${ }^{3}$ Semmes-Murphey Clinic, Memphis, Tennessee

OBJECTIVE Microendoscopic discectomy is a minimally invasive surgery technique that was initially described in 1997. It allows surgeons to work with 2 hands through a small-diameter, operating table-mounted tubular retractor, and to apply standard microsurgical techniques in which a small skin incision and minimal muscle dissection are used. Whether the surgeon chooses to use an endoscope or a microscope for visualization, the technique uses the same type of retractor and is thus called tubular microdiscectomy. The goal in this study was to review the current literature, examine the level of evidence supporting tubular microdiscectomy, and describe surgical techniques for complication avoidance.

METHODS The authors performed a systematic PubMed review using the terms "microdiscectomy trial," "tubular and open microdiscectomy," "microendoscopic open discectomy," and "minimally invasive open microdiscectomy OR microdiskectomy." Of 317 references, 10 manuscripts were included for analysis based on study design, relevance, and appropriate comparison of open to tubular discectomy.

RESULTS Similar and very favorable clinical outcomes can be expected from tubular and standard microdiscectomy. Studies have demonstrated equivalent operating times for both procedures, with lower blood loss and shorter hospital stays associated with tubular microdiscectomy. Furthermore, postoperative analgesic usage has been shown to be significantly lower after tubular microdiscectomy. Overall rates of complications are no different for tubular and standard microdiscectomy.

CONCLUSIONS Prospective randomized trials have been used to evaluate outcomes of common minimally invasive lumbar spine procedures. For lumbar discectomy, Level I evidence supports equivalently good outcomes for tubular microdiscectomy compared with standard microdiscectomy. Likewise, Level I data indicate similar safety profiles and may indicate lower blood loss for tubular microdiscectomy. Future studies should examine the comparative value of these procedures.

https://thejns.org/doi/abs/10.3171/2017.5.FOCUS17202

KEY WORDS minimally invasive spine surgery; lumbar; microdiscectomy; tubular; review; outcomes

I N 1977, Caspar ${ }^{9}$ and Yaşargi ${ }^{31}$ reported the application of microsurgical techniques to lumbar disc surgery, thus introducing the concept of microdiscectomy. By minimizing incision size and sparing paraspinal structures using a smaller, more targeted surgical exposure than traditional open discectomy, microdiscectomy served the rationale of lowering surgical approach-related morbidity, thus attempting to improve patient outcomes while retaining surgical efficacy. These principles remain the goals of all minimally invasive spine surgery. In an attempt to further these principles, microendoscopic discectomy was developed. ${ }^{11}$ This technique made use of a small-diameter tubular retractor $(14 \mathrm{~mm})$ that was placed over sequential dilators that created a surgical pathway to the lumbar spine in between fascicles of the lumbar paraspinous muscles, avoiding the traditional detachment of the multifidus muscles from the spine that is common to open discectomy and microdiscectomy. To maintain the position of the tubular retractor and free the surgeon's hands, the tube was supported by an articulated, repositionable arm that was secured to the operating table. The tube diameter was also large enough to allow 2 or 3 microsurgical instruments to be used in the surgical field simultaneously (e.g., a high-speed drill, a suction device, and a nerve root re-

ABBREVIATIONS EBL = estimated blood loss; LOS = length of hospital stay; ODI = Oswestry Disability Index; RDQ = Roland-Morris Disability Questionnaire; SF-36 = 36-Item Short Form Health Survey; VAS = visual analog scale.

SUBMITTED March 31, 2017. ACCEPTED May 11, 2017.

INCLUDE WHEN CITING DOI: 10.3171/2017.5.FOCUS17202. 
TABLE 1. Types of evidence and trials associated with the corresponding level of evidence

\begin{tabular}{cl}
\hline $\begin{array}{c}\text { Level of } \\
\text { Evidence }\end{array}$ & \multicolumn{1}{c}{ Type of Evidence } \\
\hline I & $\begin{array}{c}\text { Randomized controlled trials w/ no major limitations, } \\
\text { or systematic reviews of randomized controlled } \\
\text { trials w/ consistent findings }\end{array}$ \\
\hline II & $\begin{array}{c}\text { Prospective comparative studies, or systematic } \\
\text { reviews of either Level II studies w/ consistent find- } \\
\text { ings or Level I studies w/ inconsistent findings }\end{array}$ \\
\hline III & $\begin{array}{c}\text { Case-control studies, retrospective comparative stud- } \\
\text { ies, or systematic reviews of Level III studies }\end{array}$ \\
\hline IV & Case series \\
\hline V & Expert opinion \\
\hline
\end{tabular}

tractor). Originally, the tubular retractor was coupled with an endoscope to provide visualization. Subsequently, the operating microscope was used with the tubular retractor. Whether one uses an endoscope or a microscope for visualization, tubular microdiscectomy involves similar retractors and similar principles.

Although percutaneous lumbar spine techniques had been previously reported, they required the surgeon to use 1 hand to control a small-diameter cannula; the other hand was free, but only 1 instrument at a time could be used in the surgical field. Also, the small cannula was typically inserted into the disc through Kambin's triangle, limiting the surgeon's ability to work in the spinal canal. In contrast, tubular microdiscectomy allows the surgeon to use multiple instruments manipulated with 2 hands. It also allows the surgical cannula (tubular retractor) to be held in position without insertion into the disc space. These features enable the surgeon to perform procedures such as discectomy, laminotomy, medial facetectomy, and foraminotomy effectively, yet minimally invasively. They also allow the surgeon to address migrated disc fragments and nerve root compression due to spinal canal and foraminal stenosis.

Objectively ascribing levels of evidence to published studies is a way of categorizing available data (Tables 1-3). ${ }^{18}$ Level I represents the strongest evidence and is reserved for randomized controlled trials with no major limitations and appropriate statistical analysis, or systematic reviews of randomized controlled trials with consistent findings. Historically, Level I evidence for surgical techniques is rare. ${ }^{23}$ However, Level I evidence does exist for tubular microdiscectomy and will be described later in the paper. Level II studies are prospective comparative studies or systematic reviews of either Level II studies or Level I studies with inconsistent findings. Level III comprises case-control studies, retrospective comparative studies, or systematic reviews of Level III studies. Levels IV and V are the weakest evidence and correspond to case series and expert opinion, respectively. Appropriate synthesis of available evidence, taking into consideration the levels of evidence, can eventually lead to practice guidelines for the treatment of a specific disease process. ${ }^{15}$ The goal of this review was to summarize the data describing outcomes of one of the most widely used modalities of minimally invasive spine surgery: tubular microdiscectomy.

\section{Methods}

\section{Surgical Technique}

The senior author (K.T.F.), who invented the procedure and has performed more than 2000 tubular microdiscectomies, prefers to perform these surgeries in patients who are under general anesthesia. Although the use of local or epidural anesthesia is possible, general anesthesia has the

TABLE 2. Summary of the published literature in which tubular and standard microdiscectomy were compared with respect to demographic characteristics, operative considerations, and complications

\begin{tabular}{|c|c|c|c|c|c|c|c|c|c|}
\hline Authors \& Year & Type & No. Tubular/Standard Micro & Complications & CSF Leak & Reop & Recurrent Disc Herniation & Infection & OR Time & $\mathrm{EBL}$ \\
\hline Arts et al., 2009* & RCT & $166 / 159$ & Same & Same & Same & Same & Same & Inc & NR \\
\hline Teli et al., $2010 \dagger$ & RCT & $70 / 72$ & Same & Inc & NR & Inc & Dec & Inc & NR \\
\hline Brock et al., 2008 & $\mathrm{RCT}$ & $66 / 59$ & NR & NR & NR & NR & NR & NR & NR \\
\hline Ryang et al., 2008 & RCT & $30 / 30$ & Same & Same & Same & Same & NR & Same & Same \\
\hline Righesso et al., 2007 & RCT & $21 / 19$ & Same & Same & NR & Same & NR & $\operatorname{lnc}$ & Same \\
\hline Huang et al., 2005 & RCT & $10 / 12$ & Same & Same & NR & NR & Same & Inc & Dec \\
\hline German et al., 2008 & RCS & $49 / 123$ & NR & Same & NR & NR & NR & Same & Dec \\
\hline Lee et al., 2011 & RCS & $64 / 45$ & Same & Same & Same & Same & Same & Same & NR \\
\hline Cahill et al., 2013 & RCS & $48 / 33$ & Same & Same & NR & NR & Same & Same & NR \\
\hline Lau et al., $2011^{21}$ & RCS & $20 / 25$ & Same & Same & NR & Same & Same & Same & Dec \\
\hline
\end{tabular}

Dec = decreased; Inc = increased; micro = microdiscectomy; NR = not reported; OR time = operating time; RCS = retrospective comparative study; RCT = randomized controlled trial.

The studies are organized from the strongest first to the least strong last. The terms "increased," "decreased," and "same" denote how the tubular group compared to the standard group. These designations are based on the presence of statistically significant differences.

* Note that in the trial conducted by Arts et al., a relatively large midline incision was made for the tubular group, which deviates from the original description of the tubular procedure.

$\dagger$ The trial reported by Teli et al. included 3 groups: a tubular group, a microsurgical group, and a traditional nonmicrosurgical group. Our main interest was in the tubular and microsurgical groups, so these results are reported. 
TABLE 3. Summary of the published literature in which tubular and standard microdiscectomy were compared with respect to hospital course, short-term, and long-term outcomes

\begin{tabular}{|c|c|c|c|c|c|}
\hline Authors \& Year & $\begin{array}{l}\text { Time to } \\
\text { Mobilization }\end{array}$ & $\begin{array}{l}\text { Analgesic } \\
\text { Usage }\end{array}$ & LOS & Short-Term Outcomes & Long-Term Outcomes \\
\hline Arts et al., 2009 & Same & NR & Same & $\begin{array}{l}\text { Less improvement in RDQ, VAS leg, \& } \\
\text { VAS back scores }\end{array}$ & $\begin{array}{l}\text { Less improvement in RDQ, VAS leg, \& } \\
\text { VAS back scores }\end{array}$ \\
\hline Teli et al., 2010 & NR & NR & Inc & $\begin{array}{l}\text { Same improvement in ODI, VAS leg, } \\
\text { VAS back, \& SF-36 scores }\end{array}$ & $\begin{array}{l}\text { Same improvement in ODI, VAS leg, VAS } \\
\text { back, \& SF-36 scores }\end{array}$ \\
\hline Brock et al., 2008 & NR & Dec & NR & $\begin{array}{l}\text { Same improvement in ODI, VAS leg, \& } \\
\text { VAS back scores }\end{array}$ & NR \\
\hline Ryang et al., 2008 & NR & NR & NR & NR & Same improvement in ODI \& VAS scores \\
\hline Righesso et al., 2007 & NR & NR & Inc & $\begin{array}{l}\text { Same improvement in ODI score, less } \\
\text { improvement in VAS score at } 12 \mathrm{hrs}\end{array}$ & Same improvement in ODI \& VAS scores \\
\hline Huang et al., 2005 & NR & NR & Dec & NR & Same improvement in VAS score \\
\hline German et al., 2008 & NR & Dec & Dec & NR & NR \\
\hline Lee et al., 2011 & NR & NR & Dec & NR & NR \\
\hline Cahill et al., 2013 & NR & NR & Same & NR & NR \\
\hline Lau et al., $2011^{21}$ & NR & NR & Same & NR & NR \\
\hline
\end{tabular}

The same terminology and ordering scheme is used as in Table 2. For short- and long-term outcomes, "more improvement," "same improvement," or "less improvement" denotes more, the same, or less improvement in the tubular group compared to the standard group.

benefit of lack of patient movement during surgery and also the ability of the surgeon to obtain a full neurological examination immediately on patients' awakening, when compared with spinal anesthesia. Local anesthesia is an option; however, it can allow for patient movement during surgery, which is theoretically risky due to the fact that the retractor is fixed and bed mounted. Twenty-five manuscripts were reviewed, and 10 manuscripts were included for analysis based on study design, relevance, and appropriate comparison of open to tubular discectomy (Fig. 1).

Regardless of the anesthetic technique, tubular microdiscectomy is typically performed as an outpatient procedure. We use the Wilson frame, because it allows for suspension of the abdomen and opening of the interlaminar space. Care should be taken to position the patient as far as possible toward the head of the bed, so that the base of the operating room table does not obstruct the fluoroscopy unit in the lower lumbar region. A wide area should be prepared when draping because the incision has not yet been planned. The surgeon should stand on the side of the pathological entity, with the fluoroscopy unit brought in from the opposite side. The midline is marked by palpating the spinous processes. Occasionally, when a patient is morbidly obese and the spinous process tips are difficult to palpate, an anteroposterior fluoroscopic view may be necessary to confirm the midline. A parallel line is then drawn $15 \mathrm{~mm}$ ipsilateral to the side of the pathological entity (Fig. 2A). For morbidly obese patients and those with bulbous spinous processes, the line can be drawn a few millimeters more laterally.

The incision is planned by first inserting a 22 -gauge spinal needle through the skin along a trajectory that exactly bisects the disc space of interest (Fig. 2B). A 16-mm vertical incision is then made with the needle mark as the midpoint and is only carried down to the subcutaneous tissue. A sharp guidewire is then placed under fluoroscopic guidance along the same trajectory, piercing the dorsal lumbar fascia (Fig. 2C). An initial tubular dilator is passed over the guidewire. Once the dilator has passed through the fascia, the guidewire can be removed (Fig. 2D). The initial dilator is then used to palpate the lamina to ensure a correct docking location. The surgeon should be able to palpate anatomical landmarks such as the laminar edge and the base of the spinous process with the tip of the initial dilator. The second dilator is then passed over the initial dilator. If the fascia is dense and there is significant resistance to smooth passage of the tubular dilators, the devices can be removed and straight Mayo scissors can be placed in the fascial defect and spread in a vertical direction to dilate the fascia. The initial dilator is then replaced, followed by the second, third, and fourth (Fig. 3A and B). Once the trajectory is confirmed, a $16-\mathrm{mm}$ tubular retrac-

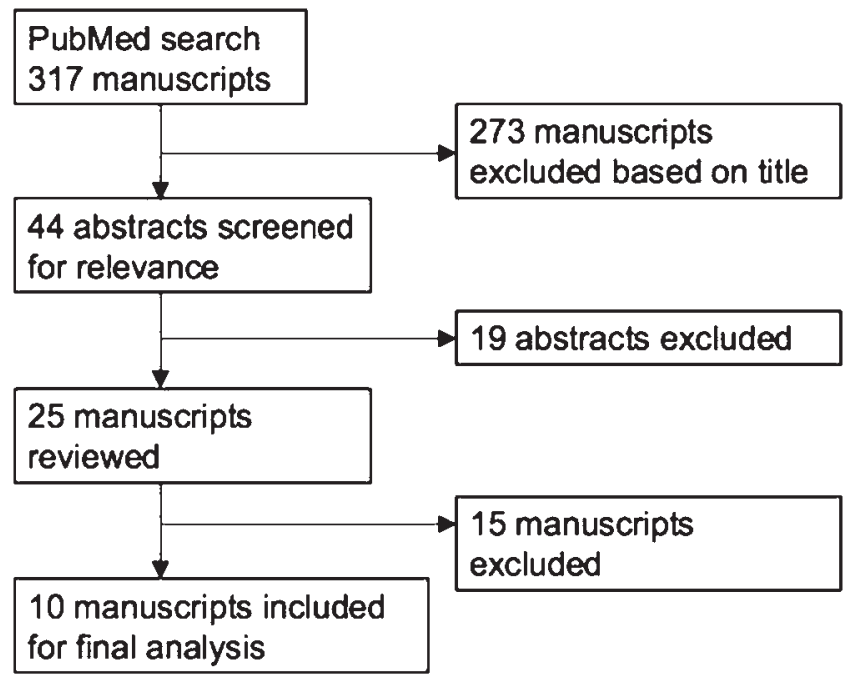

FIG. 1. The flow diagram demonstrates the search strategy, which yielded the final included manuscripts for the literature review. 

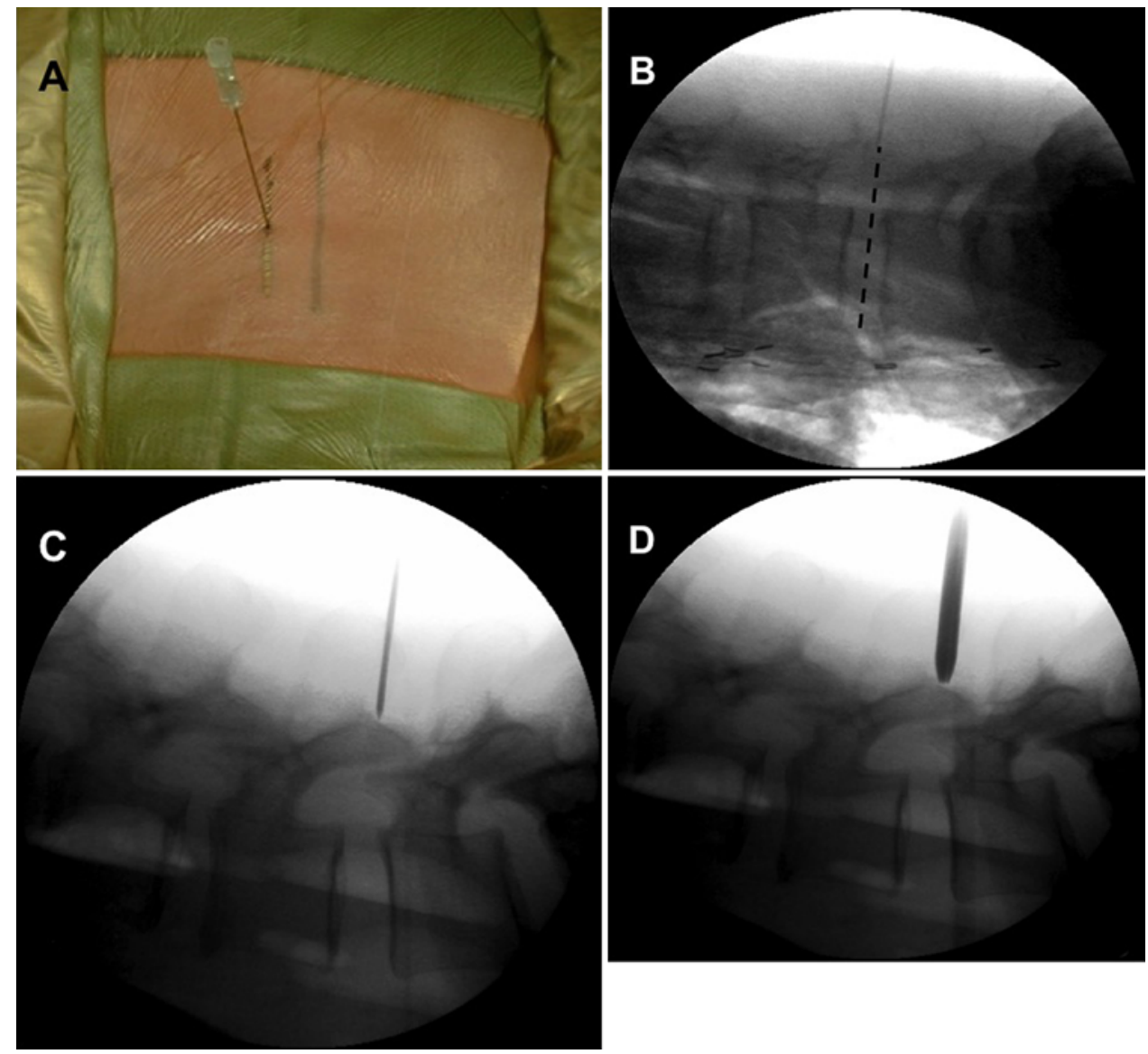

FIG. 2. Intraoperative images showing localization and muscle dilation. The midline is marked, and a spinal needle is inserted 1.5 $\mathrm{cm}$ ipsilateral to the side of the disc herniation $(\mathbf{A})$. The needle is repositioned until lateral fluoroscopy demonstrates a trajectory coaxial (dashed line) with the disc of interest (B). A sharp-tipped guidewire is placed along the same trajectory (C), followed by the first tubular dilator (D).

tor of appropriate length is placed over the fourth dilator and docked on bone (Fig. 3C and D). The flexible arm is attached to the operating room table on the side ipsilateral to the surgeon. Downward pressure applied while securing the tubular retractor prevents paraspinal muscle from entering the tube.

The operating microscope is then brought into place (Fig. 4A). Residual soft tissue can now be removed with monopolar cautery and a pituitary rongeur (Fig. 4B). Hemostasis is achieved with bipolar and/or monopolar cautery. The inferior edge of the lamina is defined and a hemilaminotomy is performed with the high-speed drill (Fig. 4C). The lamina is removed until the superior insertion of the ligamentum flavum is visualized (Fig. 4D). If necessary to gain additional exposure, the tubular retractor can be "wanded" - a process in which the largest dilator is reinserted into the tubular retractor, the flexible arm is loosened, and the retractor is angled while applying steady downward pressure to avoid unwanted soft-tissue encroachment on the operative corridor.

The ligamentum flavum is dissected free of the underlying dura mater with a modified ball dissector (Fig. 5A) and is then resected with Kerrison rongeurs (Fig. 5B). The resection should continue until the lateral edge of the traversing nerve root is visualized (Fig. 5C). A medial facetec- tomy can be performed if necessary to obtain this critical visualization. When the ball dissector can easily pass along the medial border of the inferior pedicle, the dorsal decompression is complete. The tubular retractor is then wanded until the trajectory is along the axis of the disc of interest. The nerve root retractor is placed to protect the traversing nerve root, which is retracted medially. Epidural veins are coagulated with bipolar cautery. Free disc fragments can be removed with the pituitary rongeur (Fig. 6A). Contained disc herniations are approached by incising the anulus fibrosis overlying the mass with a No. 15 bayoneted scalpel. The mass is then decompressed with a combination of straight and angled pituitary rongeurs and curettes. The decompression is complete when a ball dissector can be freely passed beneath the traversing nerve root and thecal sac without encountering a mass, and all loose fragments have been removed (Fig. 6B). An absorbable gelatin sponge soaked in $40 \mathrm{mg}$ of Depo-Medrol can be placed on an inflamed nerve root. The tubular retractor is then removed while visualizing the intact muscle fibers coming together to close the potential space. A single Vicryl (Ethicon) stitch is used to approximate the dorsal lumbar fascia. The Vicryl suture is used to close the dermis in an inverted interrupted fashion. The skin is approximated with adhesive strips. A waterproof dressing is applied so patients can shower. 

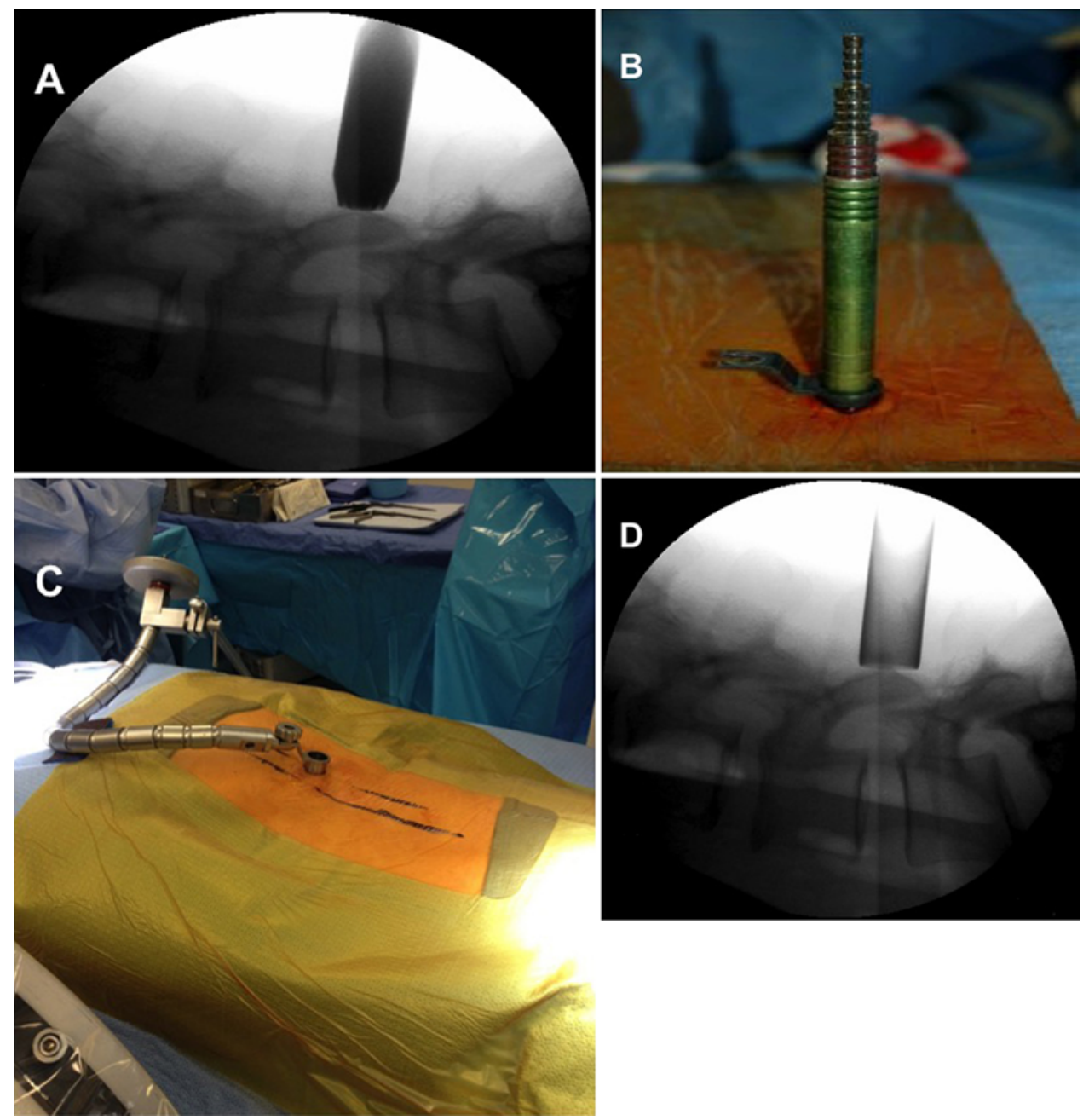

FIG. 3. Intraoperative images showing sequential dilation and tubular retractor placement. Sequentially larger dilators are placed (A), followed by the tubular retractor (B). The tubular retractor is connected to the flexible bed-mounted arm, which is positioned on the surgeon's side of the table, with the flexible arm arranged in a smooth curve to optimize stability (C). Fluoroscopy is used to confirm placement (D).

\section{Literature Review}

A comprehensive PubMed review was performed using the terms "microdiscectomy trial" and "tubular and open microdiscectomy" and "microendoscopic open discectomy" and "minimally invasive open microdiscectomy OR microdiskectomy." These searches yielded a total of 317 references. These were individually reviewed by 2 authors (A.J.C. and M.M.S.). After initial screening based on article title, 273 manuscripts were excluded. Forty-four abstracts were screened with 19 excluded based on language and relevance.

\section{Results}

\section{Operative Considerations and Complications of Tubular Discectomy}

The technique in which sequential paramedian muscle dilation and tubular retraction are used is described above, and has been subsequently adapted to use with the operating microscope. ${ }^{11}$ In 2005, the Spine Intervention Prognostic Study group conducted a randomized controlled trial to compare standard microdiscectomy with tubular discectomy. ${ }^{6}$ The Arts et al. trial evaluated short- and long-term outcomes, muscle preservation, and cost-effectiveness at 7 hospitals in the Netherlands. ${ }^{4}$ The trial was ambitious, randomizing 328 patients among the 2 groups in an intentto-treat analysis. The study was large, multicenter, doubleblinded, and statistically rigorous, and it analyzed validated outcome measures (in the form of the Roland-Morris Disability Questionnaire [RDQ] for sciatica and the visual analog scale [VAS] for back and leg pain). Furthermore, the patients were well matched at baseline, relatively few patients were excluded, and there were few missing data points throughout the study period. However, it is critical to note that the tubular operation was performed through a comparatively large $(25-30 \mathrm{~mm})$ midline incision, which represents a dramatic deviation from the initially described procedure. There were no differences in operative complications or rates of reoperation.

The trial conducted by Ryang et al. used the tubular technique as originally described and likewise demonstrated no difference in recurrent disc herniation or CSF 

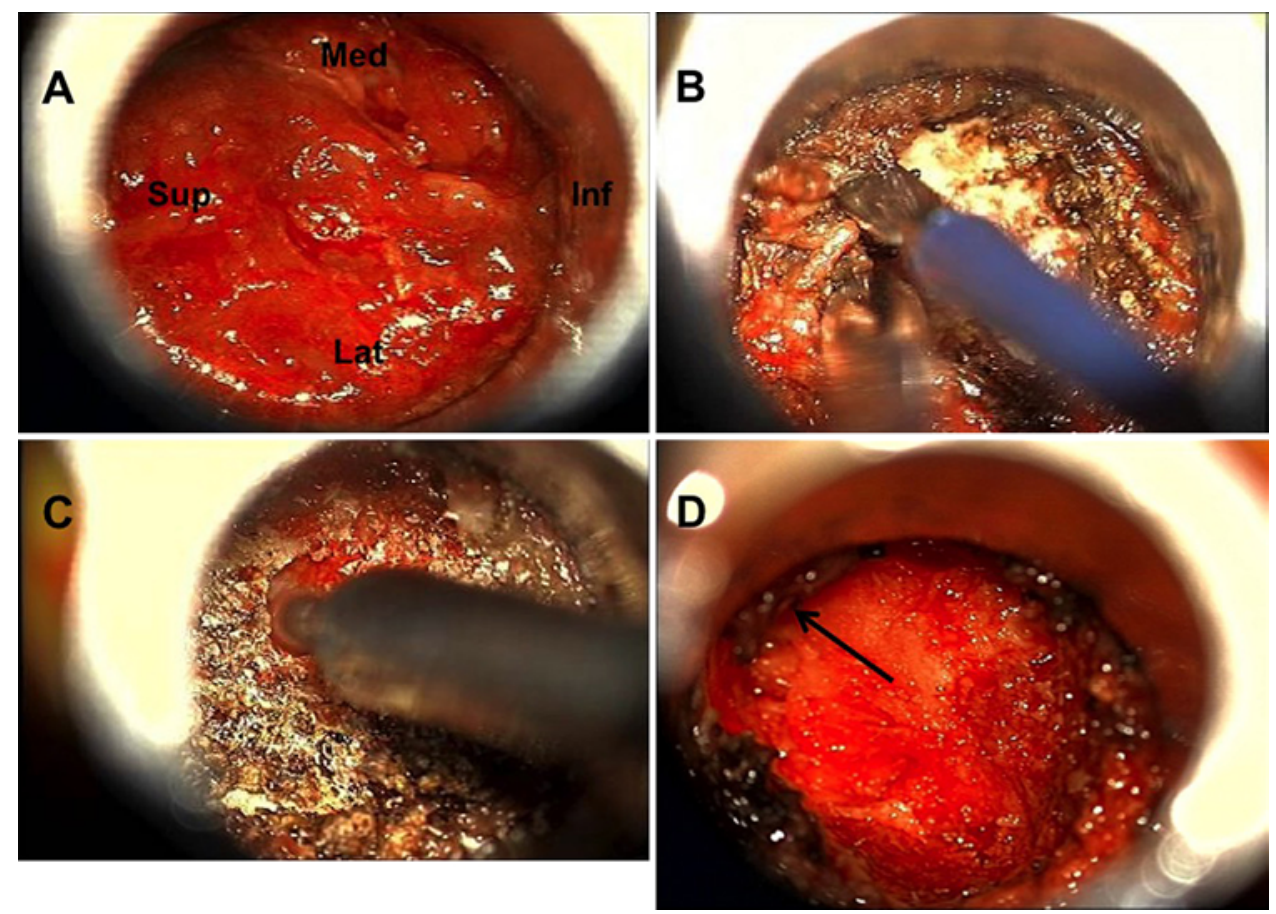

FIG. 4. Intraoperative images showing soft-tissue and bone removal. After the microscope is brought into position, residual soft tissue is visualized (A). Residual soft tissue is removed with monopolar cautery in a circumferential manner to avoid "muscle creep" (B). The lamina of the superior level is removed with the drill (C). The superior aspect of the laminotomy is the insertion of the ligamentum flavum (arrow, D). Inf = inferior; Lat = lateral; Med = medial; Sup = superior.

leaks. ${ }^{26}$ Huang et al. reported no difference in overall complications in another randomized controlled trial. ${ }^{17}$ In contrast, in a randomized controlled trial, Teli et al. demonstrated no difference in overall complications in the tubular microdiscectomy group but did demonstrate increased CSF leaks. ${ }^{29}$ Of note, no CSF leaks in the tubular group required operative revision, probably due to the largely intact paraspinal musculature, which eliminates potential space for CSF fistulas to persist. Interestingly, there were no infections in the tubular group, compared with $4 \%-5 \%$ in the standard group. Additionally, in this large trial, there was increased risk of recurrent disc herniation. Retrospective studies consistently show similar rates of overall complications and CSF leakage. ${ }^{8,13,20,22}$ Taken together, Level I evidence suggests similar safety profiles and rates of recurrence in tubular microdiscectomy compared with standard microdiscectomy.
The standard operation was associated with a statistically shorter operating time (by 11 minutes) in the trial reported by Arts et al. ${ }^{4}$ Likewise, randomized controlled trials by Righesso et al., Huang et al., and Teli et al. in which the tubular technique was used as originally described demonstrated that the standard operating time was significantly shorter than the tubular operation. ${ }^{17,25,29}$ In contrast, the trial conducted by Ryang et al. demonstrated no difference in operating times. ${ }^{26}$ Multiple retrospective studies and a meta-analysis support similar operating times for the tubular and standard groups. $8,10,13,20,22$ Therefore, Level II data suggest that operative duration may be slightly increased with tubular techniques, but this is by no means certain, because conflicting data exist.

Data on operative blood loss are also conflicting. The randomized controlled trials that specifically examined blood loss were small. The trials conducted by Ryang et
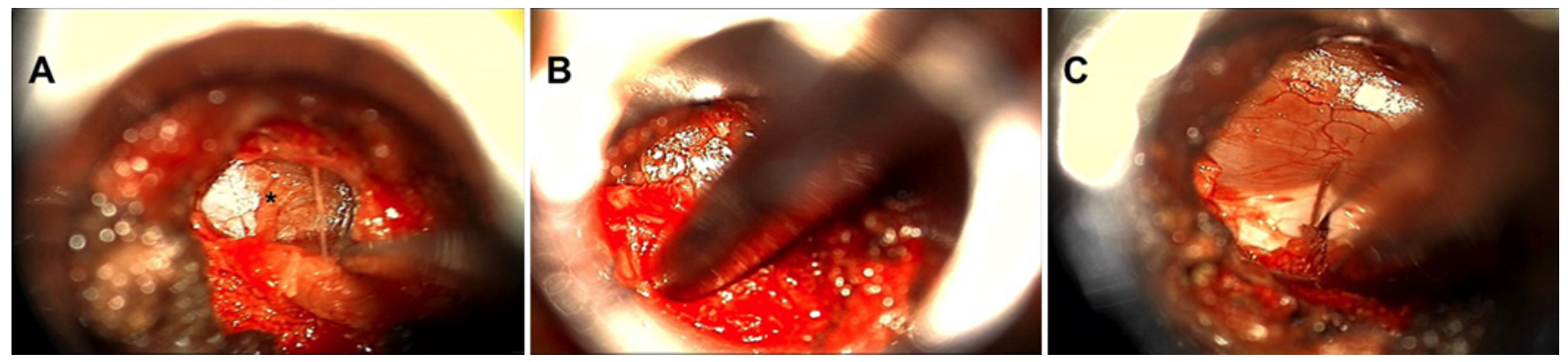

FIG. 5. Intraoperative images showing visualization of the nerve root. The ligamentum flavum is dissected using a ball-tipped dissector, with epidural fat visualized (asterisk, A). The ligamentum flavum is then resected with a Kerrison rongeur (B). If needed, a medial facetectomy is performed to visualize the lateral edge of the traversing nerve root (C). 

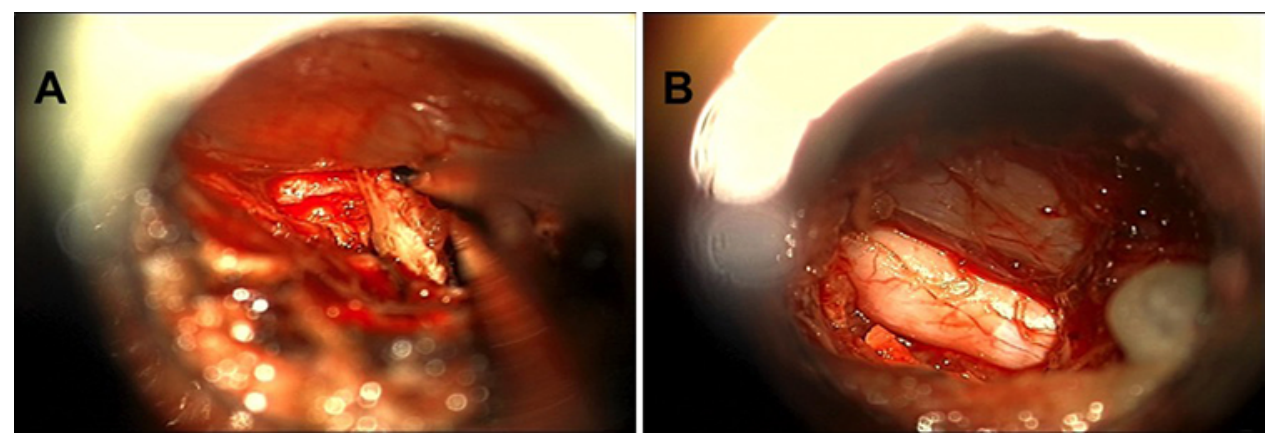

FIG. 6. Intraoperative images showing discectomy. The disc herniation is dissected free of the thecal sac and removed with a microrongeur (A). After discectomy, the nerve root is free of compression (B).

al. and Righesso et al. demonstrated no difference in estimated blood loss (EBL) between the standard and tubular groups. ${ }^{25,26}$ In contrast, the study by Huang et al. reported lower EBL in the tubular group, ${ }^{17}$ a result that is supported by retrospective studies. ${ }^{13,20}$ Level II evidence suggests that tubular microdiscectomy may be associated with similar or less blood loss than standard microdiscectomy.

\section{Perioperative, Short-Term, and Long-Term Outcomes of Tubular Microdiscectomy}

For perioperative metrics, there was no difference in time to mobilization or length of hospital stay (LOS) in the study performed by Arts et al. ${ }^{4}$ Although this may be due to differences in hospitalization patterns in the Netherlands, a US retrospective study also demonstrated no difference in the LOS. ${ }^{20}$ In contrast, 2 small randomized controlled trials showed shorter hospital stay for patients treated with tubular microdiscectomy. ${ }^{17,25}$

In the short term (8 weeks), there was no difference in RDQ or VAS scores in the study by Arts et al. ${ }^{4}$ Likewise, there were similar rates of complete recovery and time to complete recovery. During recovery, VAS leg and back and RDQ scores were slightly higher in the tubular group compared with the standard group. At 52 weeks, the RDQ score was slightly higher in the tubular group compared with the group that underwent standard microdiscectomy (4.7 vs 3.4 , respectively). Importantly, none of these differences reached the published minimally clinically important level. A follow-up manuscript by Arts et al. reported the 2-year data and demonstrated no significant differences in these outcome measures between the tubular and standard microdiscectomy groups. ${ }^{5}$ Brock et al. conducted a randomized controlled trial of standard versus tubular microdiscectomy that was more similar to the original description (although technically it was a hybrid operation because the tubular retractor used was expandable) in 125 patients. ${ }^{7}$ Follow-up was limited to the perioperative period, but demonstrated similar outcomes in Oswestry Disability Index (ODI), VAS leg, and VAS back scores. The trials by Righesso et al. and Teli et al. also demonstrated no difference in VAS or ODI scores in either group with 2 years of follow-up..$^{25,29}$ Ryang et al. demonstrated no difference in VAS, ODI, or 36-Item Short Form Health Survey (SF-36) scores at 6 months after surgery in an additional randomized controlled trial. ${ }^{26}$ Level I evidence suggests that tubular microdiscectomy is associated with outcomes similar to those of standard microdiscectomy, particularly in studies in which the procedure is used as originally described.

In the trial by Brock et al., postoperative analgesic usage was significantly lower in the tubular group, an observation that is supported by retrospective studies. ${ }^{8,13}$ Level I evidence supports lower analgesic usage in patients who undergo tubular discectomy.

Arts et al. also performed a subgroup analysis at certain larger-volume centers to evaluate changes in paraspinal musculature, and found no differences in muscle atrophy on MRI or perioperative creatine phosphokinase levels. ${ }^{3}$

\section{Cost-Effectiveness of Tubular Microdiscectomy}

In addition to demonstrating comparable results to the current gold standard, novel techniques must also be evaluated on an economic basis. Van der Akker et al. ${ }^{30}$ reported parallel cost-effectiveness results from their own study and from the trial published by Arts et al. They demonstrated no difference in total cost at 1 year between the tubular and open groups. Although costs were converted from euros to US dollars, differences between the Dutch and US health care delivery system are likely to preclude direct application. Presently, very little data exist on relative costs of tubular microdiscectomy compared with standard microdiscectomy. ${ }^{2}$ Cahill et al. retrospectively compared a series of 48 tubular microdiscectomies and 33 standard microdiscectomies. ${ }^{8}$ They reported no difference in operating room time and charges; however, total hospital charges were $\$ 5453$ less in the tubular group. They attributed this difference to a shorter LOS and lower laboratory, pharmacy, and therapy use. Therefore, Level III data indicate that tubular discectomy is associated with lower hospital charges than the standard operation in the US. Table 4 summarizes the current levels of evidence supporting these metrics.

\section{Discussion}

Our review demonstrates that complication profiles are similar for tubular microdiscectomy compared with the standard open technique. Intraoperative dural tear is the most common complication; rates range from $7 \%-$ $10 \% .4,13,20,22,29$ There are several technical nuances to avoid this frequent complication. First, once the tubular retractor is docked in the appropriate location, complete bone removal with the drill and rongeurs should be performed using the entire operative field within the tube. This bone 
TABLE 4. Level of evidence summary for tubular discectomy

\begin{tabular}{lll}
\hline \multicolumn{1}{c}{ Surgical Technique } & \multicolumn{1}{c}{ Topic } & \multicolumn{1}{c}{ Level of Evidence } \\
\hline Tubular vs open micro & Safety profiles \& recurrence & Level I: similar complications \& recurrence rates \\
\hline & Op length & Level II: duration may be slightly increased for tubular \\
\hline & EBL & Level II: EBL may be the same or slightly decreased for tubular \\
\hline & Outcomes & Level l: similar outcomes using standardized outcome measures \\
\hline & Analgesic usage & Level II: decreased analgesic usage for tubular \\
\hline Cost-effectiveness & Level III: lower hospital charges for tubular \\
\hline
\end{tabular}

decompression relieves some of the stenosis, allowing for free passage of instruments adjacent to the dura. A ball dissector should be passed under the ligamentum flavum prior to flavectomy to eliminate tethering adhesions, which could otherwise lead to durotomy. While removing the ligamentum flavum, the use of angled Kerrison rongeurs can avoid inadvertent entry of the dura into the jaws of the instrument. We prefer to use the $90^{\circ}$ rongeur when progressing medially and inferiorly, while using the $40^{\circ}$ instrument when removing tissue laterally and superiorly. Last, using the suction to provide gentle traction on the adjacent dura when removing tissue can prevent redundant dura from billowing into the instrument.

Injury to the traversing nerve root is a serious but rare intraoperative complication, with reported rates of 0\%-3\%., ${ }^{4,22,29}$ Clear identification of the relevant anatomy is required to prevent this complication. It is particularly important to define the lateral edge of the traversing root. Free passage of the ball dissector along the inferior pedicle delineates the lateral margin of bone removal. Being able to pass the ball dissector under the nerve root confirms that the lateral edge is being visualized. Recurrent disc herniation after a prior discectomy is not a contraindication for a tubular approach. ${ }^{1}$ In fact, tubular treatment of a recurrence after a standard midline approach microdiscectomy may be slightly easier than a second midline approach, because the tubular approach avoids the midline dense scar tissue from the initial operation. ${ }^{28}$ In cases of revision surgery, it is important to remove additional bone superior to the previous laminotomy to access normal dura, and then follow that plane into the scarred area.

Postoperative infection after tubular microdiscectomy is extremely uncommon, with a reported rate of $0.1 \%$ in a large prospectively collected database..$^{27}$ This is probably due to the smaller incision and lack of significant devascularized tissue and minimized dead space. Also, instruments passed into the surgical field with a tubular approach do not touch the skin edges. In the unlikely event of an infection, the case can be managed with a course of antibiotics; however, deep or persistent infections may require debridement. In our practice, we use preoperative prophylactic intravenous antibiotics and generous irrigation with antibiotic-containing sterile saline at the conclusion of the operation. Postoperative painful hematoma is a rare complication, with rates of $1 \% .{ }^{4}$ This can be avoided with the judicious use of absorbable gelatin sponge and the technique of slowly removing the tubular retractor under microscopic visualization and hemostasis with bipolar cautery. Recurrent disc herniation is a consideration after all discectomy procedures. Published rates after tubular discectomy range from $2 \%$ to $11 \%{ }^{4,21,22,29}$ Free disc fragments are removed by fragmentectomy. Contained disc herniations are removed by annulotomy and disc exploration. All loose disc material is removed. At the conclusion of discectomy, $10 \mathrm{ml}$ of sterile saline (using a $10-\mathrm{ml}$ syringe with an 8-Fr suction tip attached) is forcefully irrigated into the annulotomy site to loosen any residual tissue that would be at risk for reherniation.

With respect to clinical outcomes, studies suggest similar results in the short and long terms. As mentioned above, the largest randomized prospective study used an incision that is double the size of the normal tubular incision, as well as a midline incision instead of a paramedian one. The paramedian incision is critical, because it allows the lateral to medial trajectory necessary to minimize disruption of the multifidus muscle. In the lumbar spine, the multifidus muscle arises from the mammillary processes laterally, then runs superomedially to insert into the spinous process of the superior vertebral bodies or higher. Nevertheless, several smaller studies confirm similar rates of positive outcomes comparing tubular to open discectomy.

Of note in spine surgery, as has been demonstrated in the past, novel techniques have become the gold standard in the face of Level I evidence demonstrating equivalence to the prior widely accepted technique. Most notable is standard microdiscectomy itself. The Cochrane review has demonstrated clinical equivalence of microdiscectomy to standard open discectomy..$^{14}$ Prospective randomized studies have demonstrated similar clinical outcomes, pain medication use, complications, and hospital stay. ${ }^{16,19}$ Likewise, Level I evidence demonstrates equivalence between instrumented and noninstrumented lumbar fusion with respect to clinical improvement, complications, hospital metrics, and fusion rates..$^{12}$ These data highlight how, in the setting of equivalent outcomes, technological advances and shifting patient preferences can guide changes in treatment modalities. ${ }^{24}$

\section{Conclusions}

Prospective randomized trials have been used to evaluate outcomes of common minimally invasive lumbar spine procedures. For lumbar discectomy, Level I evidence supports equivalently good outcomes for tubular microdiscectomy compared with standard microdiscectomy. Likewise, Level I data indicate similar safety profiles, and may indicate lower blood loss for tubular microdiscectomy. Future studies should examine the comparative value of these procedures. 


\section{References}

1. Ahn J, Tabaraee E, Bohl DD, Aboushaala K, Singh K: Primary versus revision single-level minimally invasive lumbar discectomy: analysis of clinical outcomes and narcotic utilization. Spine (Phila Pa 1976) 40:E1025-E1030, 2015

2. Al-Khouja LT, Baron EM, Johnson JP, Kim TT, Drazin D: Cost-effectiveness analysis in minimally invasive spine surgery. Neurosurg Focus 36(6):E4, 2014

3. Arts M, Brand R, van der Kallen B, Lycklama à Nijeholt G, Peul W: Does minimally invasive lumbar disc surgery result in less muscle injury than conventional surgery? A randomized controlled trial. Eur Spine J 20:51-57, 2011

4. Arts MP, Brand R, van den Akker ME, Koes BW, Bartels RH, Peul WC: Tubular diskectomy vs conventional microdiskectomy for sciatica: a randomized controlled trial. JAMA 302:149-158, 2009

5. Arts MP, Brand R, van den Akker ME, Koes BW, Bartels RH, Tan WF, et al: Tubular diskectomy vs conventional microdiskectomy for the treatment of lumbar disk herniation: 2 -year results of a double-blind randomized controlled trial. Neurosurgery 69:135-144, 2011

6. Arts MP, Peul WC, Brand R, Koes BW, Thomeer RT: Costeffectiveness of microendoscopic discectomy versus conventional open discectomy in the treatment of lumbar disc herniation: a prospective randomised controlled trial [ISRCTN51857546]. BMC Musculoskelet Disord 7:42, 2006

7. Brock M, Kunkel P, Papavero L: Lumbar microdiscectomy: subperiosteal versus transmuscular approach and influence on the early postoperative analgesic consumption. Eur Spine J 17:518-522, 2008

8. Cahill KS, Levi AD, Cummock MD, Liao W, Wang MY: A comparison of acute hospital charges after tubular versus open microdiskectomy. World Neurosurg 80:208-212, 2013

9. Caspar W: A new surgical procedure for lumbar disc herniation causing less tissue damage through a microsurgical approach. Adv Neurosurg 4:74-80, 1977

10. Dasenbrock HH, Juraschek SP, Schultz LR, Witham TF, Sciubba DM, Wolinsky JP, et al: The efficacy of minimally invasive discectomy compared with open discectomy: a meta-analysis of prospective randomized controlled trials. J Neurosurg Spine 16:452-462, 2012

11. Foley KT, Smith MM: Microendoscopic discectomy. Tech Neurosurg 3:301-307, 1997

12. Fritzell $P$, Hägg $\mathrm{O}$, Wessberg $\mathrm{P}$, Nordwall A: Chronic low back pain and fusion: a comparison of three surgical techniques: a prospective multicenter randomized study from the Swedish lumbar spine study group. Spine (Phila Pa 1976) 27:1131-1141, 2002

13. German JW, Adamo MA, Hoppenot RG, Blossom JH, Nagle HA: Perioperative results following lumbar discectomy: comparison of minimally invasive discectomy and standard microdiscectomy. Neurosurg Focus 25(2):E20, 2008

14. Gibson JN, Waddell G: Surgical interventions for lumbar disc prolapse: updated Cochrane Review. Spine (Phila Pa 1976) 32:1735-1747, 2007

15. Hayward RS, Laupacis A: Initiating, conducting and maintaining guidelines development programs. CMAJ 148:507512,1993

16. Henriksen L, Schmidt K, Eskesen V, Jantzen E: A controlled study of microsurgical versus standard lumbar discectomy. Br J Neurosurg 10:289-293, 1996

17. Huang TJ, Hsu RW, Li YY, Cheng CC: Less systemic cytokine response in patients following microendoscopic versus open lumbar discectomy. J Orthop Res 23:406-411, 2005

18. Kaiser MG, Eck JC, Groff MW, Watters WC III, Dailey AT, Resnick DK, et al: Guideline update for the performance of fusion procedures for degenerative disease of the lumbar spine. Part 1: introduction and methodology. J Neurosurg Spine 21:2-6, 2014
19. Katayama Y, Matsuyama Y, Yoshihara H, Sakai Y, Nakamura H, Nakashima S, et al: Comparison of surgical outcomes between macro discectomy and micro discectomy for lumbar disc herniation: a prospective randomized study with surgery performed by the same spine surgeon. J Spinal Disord Tech 19:344-347, 2006

20. Lau D, Han SJ, Lee JG, Lu DC, Chou D: Minimally invasive compared to open microdiscectomy for lumbar disc herniation. J Clin Neurosci 18:81-84, 2011

21. Lau D, Lee JG, Han SJ, Lu DC, Chou D: Complications and perioperative factors associated with learning the technique of minimally invasive transforaminal lumbar interbody fusion (TLIF). J Clin Neurosci 18:624-627, 2011

22. Lee P, Liu JC, Fessler RG: Perioperative results following open and minimally invasive single-level lumbar discectomy. J Clin Neurosci 18:1667-1670, 2011

23. Mannion R: Spine surgery: Minimally invasive spinal surgery-does size matter? Nat Rev Neurol 8:363-365, 2012

24. Oppenheimer JH, DeCastro I, McDonnell DE: Minimally invasive spine technology and minimally invasive spine surgery: a historical review. Neurosurg Focus 27(3):E9, 2009

25. Righesso O, Falavigna A, Avanzi O: Comparison of open discectomy with microendoscopic discectomy in lumbar disc herniations: results of a randomized controlled trial. Neurosurgery 61:545-549, 2007

26. Ryang YM, Oertel MF, Mayfrank L, Gilsbach JM, Rohde V: Standard open microdiscectomy versus minimal access trocar microdiscectomy: results of a prospective randomized study. Neurosurgery 62:174-182, 2008

27. Shousha M, Cirovic D, Boehm H: Infection rate after minimally invasive noninstrumented spinal surgery based on 4350 procedures. Spine (Phila Pa 1976) 40:201-205, 2015

28. Smith JS, Ogden AT, Shafizadeh S, Fessler RG: Clinical outcomes after microendoscopic discectomy for recurrent lumbar disc herniation. J Spinal Disord Tech 23:30-34, 2010

29. Teli M, Lovi A, Brayda-Bruno M, Zagra A, Corriero A, Giudici F, et al: Higher risk of dural tears and recurrent herniation with lumbar micro-endoscopic discectomy. Eur Spine J 19:443-450, 2010

30. van den Akker ME, Arts MP, van den Hout WB, Brand R, Koes BW, Peul WC: Tubular diskectomy vs conventional microdiskectomy for the treatment of lumbar disk-related sciatica: cost utility analysis alongside a double-blind randomized controlled trial. Neurosurgery 69:829-836, 2011

31. Yasargil MG: Microsurgical operations of herniated lumbar disc. Adv Neurosurg 4:81-82, 1977

\section{Disclosures}

Dr. Foley is a consultant for, owns stock in, and is a patent holder with Medtronic. He also owns stock in NuVasive, SpineWave, and Discgenics.

\section{Author Contributions}

Conception and design: Clark, Foley. Acquisition of data: Clark, Safaee, Khan. Analysis and interpretation of data: Clark, Safaee, Khan, Foley. Drafting the article: all authors. Critically revising the article: all authors. Reviewed submitted version of manuscript: all authors. Approved the final version of the manuscript on behalf of all authors: Clark. Statistical analysis: Clark, Foley. Administrative/technical/material support: Clark, Foley. Study supervision: Clark, Foley.

\section{Correspondence}

Aaron J. Clark, Department of Neurological Surgery, University of California, San Francisco, 400 Parnassus Ave., 3rd Fl., San Francisco, CA 94143-0332. email: aaron.clark@ucsf.edu. 